\title{
A rare case of a patient with a foreign body in the esophagus for two years which perforated into the mediastinum
}

\author{
*Byaruhanga $\mathrm{R}^{1}$, Kakande $\mathrm{E}^{1}$, Mwambu $\mathrm{T}^{2}$
}

1. Department of Otolaryngology, School of Medicine, College of Health Sciences, Makerere University, Kampala, Uganda.

2. Fellow ICVD Cardiothoracic Surgery, Department of Surgery, Cardiovascular Division, Mulago National Referral Hospital, Kampala, Uganda.

\begin{abstract}
A 6-year-old girl was referred to the ENT (Ear nose and throat) unit at Mulago National Referral Hospital with a foreign body in the esophagus diagnosed by routine chest radiograph. The child's parents recall she had ingested a round object (galvanised iron umbrella cap of a roofing nail) two years prior to this, but they thought that the child had passed it out in stool since she had continued eating and swallowing normally.

On arrival at the National referral hospital, the child had two esophagoscopies done but the foreign body was not seen, not until a barium swallow was done was it confirmed that the $\mathrm{FB}$ (foreign body) had perforated the esophagus and entered the mediastinum.

The cardiothoracic surgeons were consulted, and they removed the foreign body via a thoracotomy.

The child recovered well and was discharged from hospital on day 55 .

African Health Sciences 2012; (4): 569 - 571 http://dx.doi.org/10.4314/ahs.v12i4.28
\end{abstract}

\section{Introduction}

A 6-year-old girl was referred to the ENT(Ear, nose and throat) unit at Mulago National Referral Hospital for a foreign body in the esophagus diagnosed by routine chest radiograph. The child's radiograph was performed following a two week history of cough and a four week history of dysphagia. On taking historyfrom the child's parents, it was noted that she had ingested a metallic foreign body two years earlier. It had been assumed that this foreign body had been passed in her stool and the child was not investigated at that time.

Her physical examination revealed a child who was afebrile with a productive cough. Her vital signs were normal. She was brought to the operating room for a rigid esophagoscopy with removal of foreign body by the ENT resident. No foreign body was identified on this initial esophagoscopy. The following day a consultant ENT surgeon brought the patient back for a repeat rigid esophagoscopy but once again no foreign body was identified.

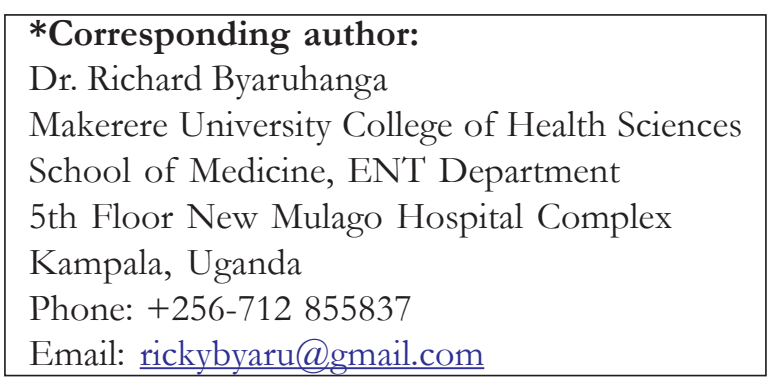

The ENT service then organized for the patient to undergo a water-soluble contrast barium swallow. This demonstrated that the foreign body was posterior to the esophagus and a small fistula had been created at the junction of the upper third and mid third esophagus which led blindly to the mediastinum(figure 1). A CT(Computerised tomograghy) scan of the chest confirmed that the $\mathrm{FB}$ was present in the right mediastinal area superior to the carina.

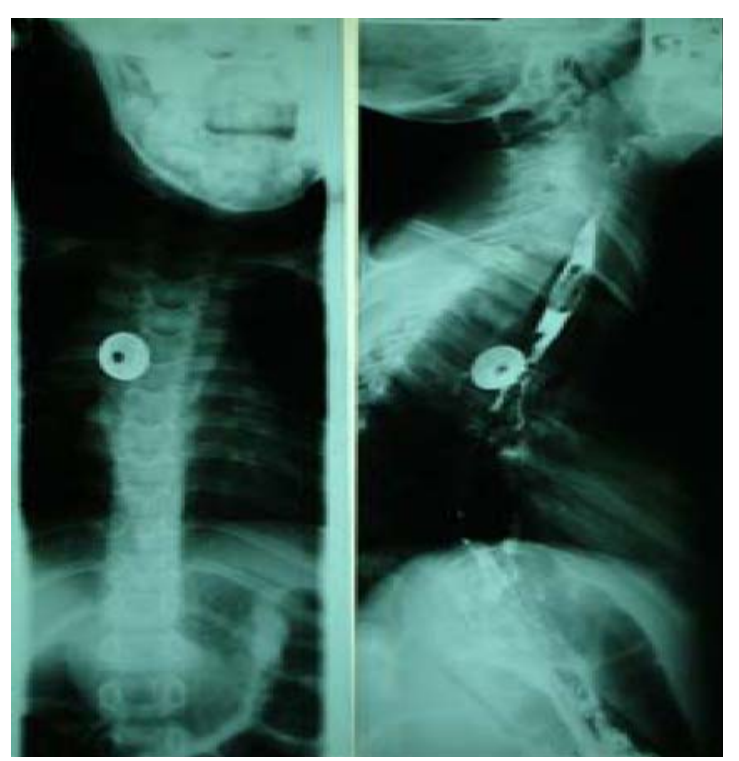

Figure 1: Barium swallow showing FB in mediastinum 
A cardiothoracic team consult was obtained. Under general anaesthesia given via an endotracheal tube, a right thoracotomy and posterior mediastinal exploration using a right thoracotomy incision was performed on admission day seven. A portion of the posterior mediastinal wall was found to be thickened corresponding to approximately $5^{\text {th }}$ thoracic spine. Adjacent apical lung tissues had reduced compliance/aeration, marked membraneous adhesions. No effusion was seen.

The mediastinum was then opened, and a black round foreign body was found in a cavity of granulomatous tissues. The foreign body was removed, the cavity tissues curretted and the chest lavaged. A chest tube connected to underwater seal drainage and NG tube were inserted and haemostasis was achieved. The lungs were then expanded and the wound closed in layers. Suffice to say that induction, maintainance and reversal of the general anaesthetic was smooth and uneventful.

Postoperatively, she was admitted spontaneously breathing without endotracheal tube to the ICU for monitoring. She developed right lower lobe pneumonia and was placed on the following antibiotics metronidazole, ampicillin, Gentamycin. She also received Pethidine, and Diclofenac and later paracetamol. Chest physiotherapy was also done daily. Three days after being admitted to ICU she was transferred to the floor. In the first 14 days postoperative, the patient was taught how to do chest physiotherapy by blowing a balloon in order to help expand her lungs which she did under the strict supervision of her parents. Thirty days after the operation, a repeat water-soluble contrast swallow was done and results revealed that the sinus in the oesophagus had closed. The NG tube was then removed and child started feeding orally starting with oral sips. On admission day 55 she was discharged home in good condition.

\section{Discussion}

Ingested foreign body is a common clinical problem in Uganda. Our hospital data (Mulago hospital, ENT dept data) shows that the commonest foreign bodies in the esophagus are coins followed by fish bones Esophageal perforations as a complication resulting from these ingested foreign bodies are rare occurences, and sometimes are iatrogenically caused. The incidence of esophageal perforation following foreign-body ingestion in some studies has been found to be $0.21 \%{ }^{1}$ and in another study was $0.96 \% \%^{2}$. Naidoo et al from Durban, South Africa also reported two cases of esophageal perforations frollowing chronic retained foreign bodies ${ }^{3}$.
When a perforation occurs, it is associated with significant morbidity and mortality. It is accepted that a cervical perforation carries a better prognosis than a thoracoabdominal perforation ${ }^{4,5,6}$, and an early diagnosis, especially within 24 hours has been associated with a better prognosis in most series ${ }^{4,7,8-}$ ${ }^{10}$. In our case, the girl had swallowed the foreign body two years prior to admission and was seen in hospital two weeks after she had started getting symptoms.

Most authors suggest early surgical closure of the esophageal fistula and drainage of the contaminated area $^{4,11,12}$. However, good results have also been reported with conservative approaches ${ }^{13-15}$. Our patient had a thoracotomy done on her a week after arriving in hospital, and the foreign body was removed, but the fistula was not repaired and was left to heal on its own with an NG tube insitu. Although she experienced some postoperative infection, she finally healed well and the fistula closed.

Most esophageal perforations occur with sharp foreign bodies. In this case the foreign body was round and happened to lodge itself in the esophagus. The foreign body remained lodged for 2 years post ingestion. Eventually it migrated to the mediastinum and became symptomatic.

\section{Conclusion}

From the above case we suggest that if a patient reports to an ENT referral unit with a history of a foreign body ingestion, and symptoms and signs suggestive of probable perforation of the FB into the esophagus, the first esophagoscopy to be done should be done by an experienced ENT team( rather than a resident doing it alone).

Medical officers at peripheral units should be advised that upon seeing any patient with a history of having ingested a foreign body with or without symptoms should be investigated with a baseline chest xray before discharging them. This is to prevent delays in making a diagnosis and risks of perforation.

\section{References}

1. Chuen KHL, Woo KSJ, Hasselt CA. Esophageal perforation and neck abscess from ingested foreign bodies: treatment and outcomes Ear, Nose \& Throat Journal Oct, 2003

2. Nandi P, Ong GB. Foreign body in the oesophagus: Review of 2394 cases. BrJ Surg 1978;65:5-9.

African Health Sciences Vol 12 Issue 4 December 2012 
3. Naidoo RR, Reddi AA. Chronic retained foreign bodies in the esophagus. Ann Thorac Surg. 2004 Jun;77(6):2218-20.

4. Sawyers JL, Lane CE, Foster JH, Daniel RA. Esophageal perforation: An increasing challenge. Ann Thorac Surg 1975;19: 233-8.

5. Bladergroen MR, Lowe JE, Postlethwait RW. Diagnosis and recommended management of esophageal perforation and rupture. Ann Thorac Surg 1986;42:235-9.)

6. Barrett NR. Report of a case of spontaneous perforation of the oesophagus successfully treated by operation. Br J Surg 1947; 35:21618.

7. Silvis SE, Nebel O, Rogers G, et al. Endoscopic complications. Results of the 1974 American Society for Gastrointestinal Endoscopy Survey. JAMA 1976;235:928-30.

8. Skinner DB, Little AG, DeMeester TR. Management of esophageal perforation. $\mathrm{Am} \mathrm{J}$ Surg 1980;139:760-4.
9. Richardson JD, Martin LF, Borzotta AP, Polk HC, Jr. Unifying concepts in treatment of esophageal leaks. Am J Surg 1985;149: 157-62.

10. Michel L, Grillo HC, Malt RA. Operative and non-operative management of esophageal perforations. Ann Surg 1981;194: 57-63.

11. Tulman AB, Boyce HW, Jr. Complications of esophageal dilation and guidelines for their prevention. Gastrointest Endosc 1981; 27:229-34.

12. Nesbitt JC, Sawyers JL. Surgical management of esophageal perforation. Am Surg 1987;53:183-91.

13. Wesdorp IC, Bartelsman JF, Huibregtse K, et al. Treatment of instrumental oesophageal perforation. Gut 1984;25:398-404.

14. Cameron JL, Kieffer RF, Hendrix TR, et al. Selective non-operative management of contained intrathoracic esophageal disruptions. Ann Thorac Surg 1979;27:404-8.

15. Shaffer HA, Jr., Valenzuela G, Mittal RK. Esophageal perforation. A reassessment of the criteria for choosing medical or surgical therapy. Arch Intern Med 1992;152:757-61. 